\title{
Undetectable $S$ cone electroretinogram b-wave in complete congenital stationary night blindness
}

\author{
Megumi Kamiyama, Shuichi Yamamoto, Koji Nitta, Seiji Hayasaka
}

\begin{abstract}
Aims-The short wavelength sensitive (S) cone electroretinograms (ERGs) were examined in two patients with the complete type of congenital stationary night blindness (CSNB).

Methods-Ganzfeld spectral flashes in the presence of strong white adapting fields were used to elicit the $S$ cone ERGs.

Results-The S cone ERG b-wave was not detectable to short wavelength stimuli, while the mixed long (L) and middle (M) wavelength sensitive cone responses appeared normal in waveforms with normal amplitude in both patients. Both patients had normal colour vision on the Farnsworth Panel D-15.

Conclusions-These ERG results indicated that the $S$ cone system as well as rod system is more impaired in complete CSNB than the $L$ and $M$ cone system and that normal colour vision may not depend on a normal $S$ cone ERG to full field stimuli.

(Br F Ophthalmol 1996;80:637-639)
\end{abstract}

The Schubert-Bornschein type ${ }^{1}$ of congenital stationary night blindness (CSNB), in which a negative electroretinogram (ERG) is typically observed and the presence of an abnormality in neural transmission at the outer retina is proposed, ${ }^{2}$ has been divided into a complete type and an incomplete type by Miyake and associates. ${ }^{3}$ In this report, we describe two patients with the complete type of CSNB, whose short wavelength sensitive (S) cone ERGs were tested to ganzfeld stimuli. The $S$ 4 April 1996 cone ERG b-wave to short wavelength stimuli was absent in both patients.

\section{Patients and methods}

REPORT OF CASES

Case 1

A 5-year-old boy has complained of visual disturbance and night blindness. There was no consanguinity. The parents and the patient's younger brother had normal visual function. The visual acuity was $20 / 30$ with a correction of -1.5 dioptres in both eyes. Ophthalmoscopic examination showed normal findings except for slight pallor of the temporal side of both optic discs. He had normal colour vision on the Farnsworth Panel D-15. The 100 Hue test was unable to be done because of his young age. Rod dark adaptation tested by a GoldmannWeekes adaptometer was absent and the cone adaptation showed an elevated final threshold (Fig 1).

Case 2

A 12-year-old boy also complained of visual disturbance and night blindness of long duration. His parents were first cousins. No ophthalmic abnormality was reported. His visual acuity was $20 / 40$ in the right eye and $20 / 25$ in the left, with a correction of -7.5 dioptres in both eyes. On ophthalmoscopic examination, tigroid fundi were seen in both eyes. Dark adaptometry showed only cone function with an elevated final threshold (Fig 1). Colour vision tested by the Farnsworth Panel D-15 was normal; however, the results of the 100 Hue test were not repeatable probably due to his age.

\section{ELECTRORETINOGRAPHY}

The method for ERG recordings was almost identical to that of Gouras et al. ${ }^{4}$ The pupils were fully dilated with tropicamide eyedrops, and the ERG was recorded simultaneously from both eyes using the Burian-Allen contact lens electrodes (Hansen Ophthalmics, Iowa City, IA, USA). A Neuropack 2 averager (Nihon Kohden, Tokyo, Japan) in combination with a ganzfeld stimulator, which provided full field flash stimuli and white background illumination, was used for stimulation and recording. A single bright flash ERG was recorded after 30 minutes of dark adaptation. Cone ERG was recorded to white stimuli presented at $5 \mathrm{~Hz}$ under white background light $\left(50 \mathrm{~cd} / \mathrm{m}^{2}\right)$. Rod responses were recorded to dim blue flashes presented at $1 \mathrm{~Hz}$ after 30 minutes of dark adaptation. Spectral stimuli 
Single flash ERG Cone ERG Rod ERG

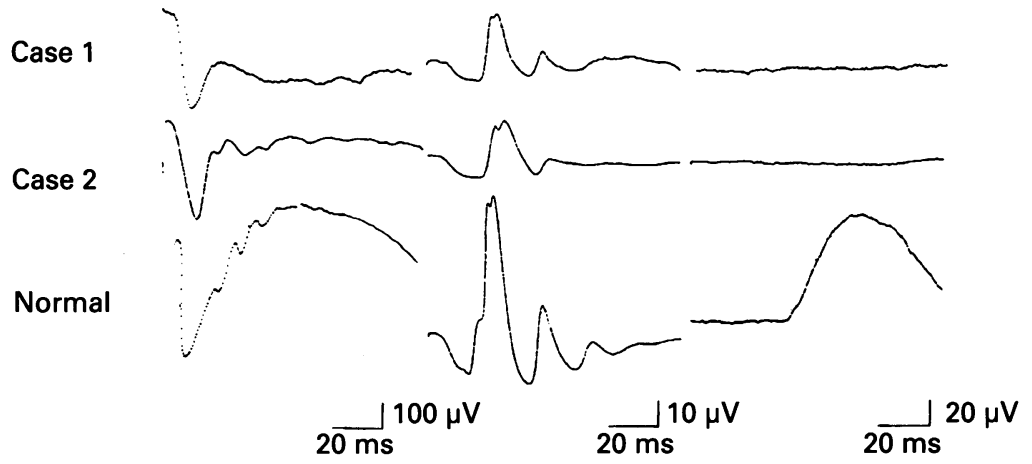

Figure 2 Single bright flash ERGs (left), cone ERGs to white flashes in the presence of a white adapting light (middle), and rod ERGs to dim blue flashes after 30 minutes of dark adaptation (right) from a 10-year-old normal subject and two patients with cogenital stationary night blindness.

were produced by Kodak Wratten colour filters (Eastman Kodak Co, Rochester, NY, USA), 98 $(450 \mathrm{~nm}), 48(471 \mathrm{~nm}), 61(534 \mathrm{~nm}), 21$ (593 $\mathrm{nm})$, and $29(633 \mathrm{~nm})$, on the same white background illumination. The stimulus frequency was $5 \mathrm{~Hz}$ and responses were averaged 500 times.

\section{Results}

A single bright flash ERG showed a negative type ERG in both patients (Fig 2). The cone ERG a-wave showed a squared off appearance, and the b-wave amplitude was below normal limits (Fig 2). Rod responses to dim blue flashes were non-recordable (Fig 2).

In normal subjects, the $S$ cone ERG, elicited by short wavelength flashes (450 and $471 \mathrm{~nm}$ ), appeared as a separate b-wave riding on an earlier b-wave of the mixed $L$ and $M$ cone ERGs (Fig 3). The mean (SD) for $S$ cone b-wave amplitude in 25 subjects aged from 9 years to 30 years is $1.6(0.6) \mu \mathrm{V}$ in our laboratory . To middle and long wavelength stimuli, only the L-M cone b-waves appeared. In both cases, the L-M cone responses were elicited to 450 and $471 \mathrm{~nm}$ stimuli, which were almost identical to those produced with longer wavelength stimuli. However, both patients had no detectable $S$ cone response to short wavelength stimuli and only a distinct negative wave appeared after the L-M cone b-wave (Fig 3).
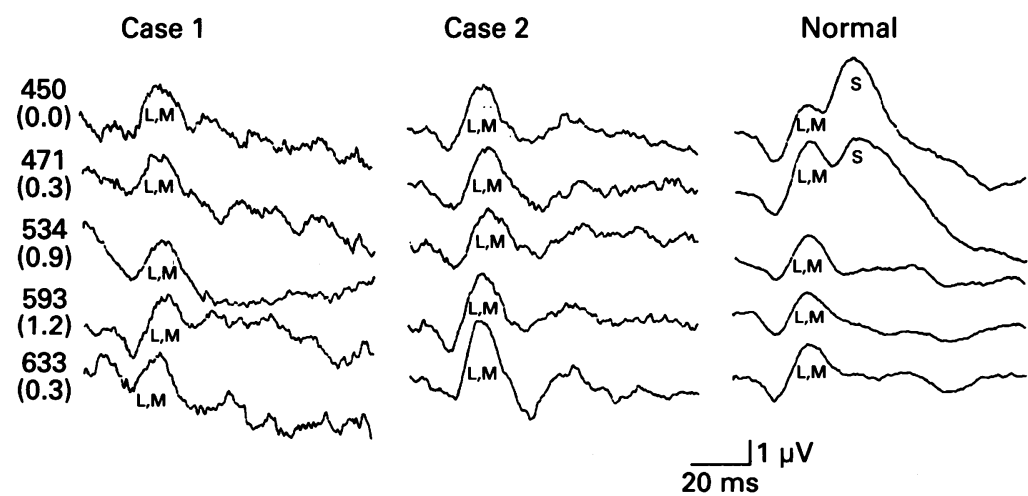
Figure 3 Cone ERGs to spectral stimuli from our patients (left and middle) and a normal
subject (right) were obtained in the presence of a white adapting light. The numbers on the left signify the wavelengths of the stimuli. Numbers in parentheses indicate the neutral density filter used to adjust the energies of the flashes to produce approximately equal early (L-M cone) b-waves.

\section{Discussion}

Our patients demonstrated a negative shaped ERG to a single bright flash, absence of rod function measured electrophysiologically and psychophysically, moderately poor visual acuity, and normal fundus appearance except mild myopic changes. These clinical features in our patients were quite similar to those reported previously in the complete type of CSNB. ${ }^{3}$

The cone ERG to white stimuli in the presence of bright white illumination showed squared off appearance of the a-wave due to the absence of the oscillatory potentials. The cone ERG b-wave was slightly reduced in amplitude with normal implicit time. These properties of the cone ERG in CSNB have been reported previously. ${ }^{5-7}$ To our knowledge, however, $\mathrm{S}$ cone function was previously evaluated only by MacKay and associates. ${ }^{8}$ In our patients, only the L-M cone b-wave was elicited to our maximum blue flashes, with normal amplitude and normal implicit times. The $S$ cone b-wave, which appeared as a separate b-wave just after an early L-M cone b-wave in the normal control, was completely absent. Any trace of the $S$ cone b-wave was not detected and a distinct negative wave appeared just after the L-M cone b-wave. To longer wavelength stimuli, the L-M cone responses appeared normal and the waveforms were quite similar to those to blue flashes. These ERG findings for different chromatic stimuli indicated that the $S$ cone mechanism is selectively affected compared with the L-M cone ERG. Our results were identical to those of MacKay et al. . $^{8}$

The rod system has only 'on' bipolars and involves an intermediary amacrine cell pathway. The $S$ cone pathway is less well known but there has been some evidence that there is preponderance of S cone 'on' units at the ganglion cell level. ${ }^{9}$ The defect in the complete type of CSNB may involve the 'on' pathway exclusively ${ }^{10}$ and therefore have a more profound effect on both the $S$ cone and rod bipolar systems. The cone ERGs to white stimuli were also deteriorated in our patients, because the 'on' pathway of the L-M cone system was defective.

It is well known that the $S$ cone system is vulnerable in retinal disease. The $S$ cone ERG was reportedly unrecordable in patients with congenital tritanopia ${ }^{11}$ and was reduced but not absent in subjects heterozygous for a defect in the $S$ cone opsin gene. ${ }^{12}$ Patients with retinitis pigmentosa also showed greater loss in the $S$ cone ERG than in the L-M cone ERG. ${ }^{13}$ Recently, the authors reported that the $S$ cone ERG was not detectable in a case of crystalline retinopathy, whereas the L-M cone b-wave were recordable although reduced. ${ }^{14}$

MacKay and associates have reported that the $S$ cone ERG may depend upon the presence of normal rod function and may not be essential for normal colour vision. ${ }^{8}$ Interestingly, in spite of non-recordable $S$ cone b-waves, our patients' colour vision tested with the Farnsworth Panel D-15 was also normal. This test is known to be very insensitive and other psychophysical tests may be necessary for 
the evaluation of our patients' colour vision. Milam et $a l^{15}$ tested $S$ cone perimetry in two patients with CSNB and reported normal $S$ cone sensitivity at fixation and at a few paracentral loci, and reduced function at $60 \%$ of the test loci. However, the S cone ERG was not tested in their patients. Our patients' $S$ cone function may be normal at fixation and impaired at the periphery, and this may be the reason for normal colour vision with the absent $S$ cone ERG. Further studies are needed to clarify the relation between the full field $S$ cone ERG and colour vision.

The authors are grateful to Peter Gouras, MD, for helpful comments on the manuscript. This study was supported in part by a grant from the Japanese Ministry of Education, Science and Grant from

1 Schubert G, Bornschein H. Beitrag zur Analyse des menschlichen Electroretinogram. Ophthalmologica 1952;123:396-413.

2 Ripps H. Night blindness revisited: from man to molecules. Invest Ophthalmol Vis Sci 1982;23:588-609.

3 Miyake Y, Yagasaki Y, Horiguchi M, Kawase Y, Kanda T. Congenital stationary night blindness with negative coctiotin: 1986;104:1013-20.

4 Gouras P. MacKay CJ, Yamamoto S. The human S-cone electroretinogram and its variation among subjects with and without $\mathrm{L}$ and $\mathrm{M}$-cone function. Invest OphthalmolVis Sci 1993;34:2437-42.

5 Krill AE, Martin D. Photopic abnormalities in congenital stationary nightblindness. Invest Ophthalmol 1971;10:62536.

6 Heckenlively JR, Martin DA, Rosenbaum AL. Loss of electroretinographic oscillatory potentials, optic atrophy, and dysplasia in congenital stationary night blindness. $\mathrm{Am} \mathcal{F}$ Ophthalmol 1983;96:526-34.

7 Lachapelle P, Little JM, Polomeno RC. The photopic electroretine $\mathrm{P}$, Little JM, Polomeno RC. The photopic electroretinogram in congenital stationary night blindn

8 MacKay CJ, Saeki M, Gouras P, Roy M. Congenital and acquired nyctalopia eliminate the S-cone ERG without disturbing color vision. Invest Ophthalmol Vis Sci 1995, 36(4,suppl):S925.

$9 \mathrm{Kolb}$ H, Lipetz LE. The anatomical basis for colour vision in the vertebrate retina. In: Gouras P, ed. The perception of colour. London: MacMillan Press, 1991:128-45.

10 Miyake Y, Yagasaki K, Horiguchi M, Kawase Y. On- and offresponses in photopic electroretinogram in complete and respoms te fpn $\mathcal{F}$ Ophthalmol 1987;31:81-7.

11 Miyake Y, Yagasaki K, Ichikawa $H$. Differential diagnosis of congenital tritanopia and dominantly inherited juvenile congenital tritanopia and dominantly inherited juvenic atrophy. Arch Ophthalmol 1985;103:1496-501.
optic

12 Bailey JE, Montag E. Short wavelength sensitive cone system function in hereditary tritanopia. Invest Ophthalmo Vis Sci 1992;33(4,Suppl):701.

13 Swanson WH, Birch DG, Anderson JL. S-cone function in patients with retinitis pigmentosa. Invest OphthalmolVis $S_{c}$ 1993;34:3045-55.

14 Yamamoto S, Kataoka Y, Kamiyama M, Hayasaka S. Non-detectable S-cone electroretinogram in a patient with crystalline retinopathy. Doc Ophthalmol 1995;90:221-7.

15 Milam AH, Saari JC, Jacobson SG, Lubinski WP, Feun LG Alexander KR. Autoantibodies against retinal bipolar cells in cutaneous melanoma-associated retinopathy. Invest $O p h$ in cutaneous melanoma-associated 